\title{
SPECIFYING MANUFACTURING TOLERANCES FOR A GIVEN AMPLIFICATION FACTOR: A NONPARAMETRIC PROBABILISTIC METHODOLOGY
}

\author{
Evangéline Capiez-Lernout \\ Laboratory of Engineering Mechanics (LaM) \\ University of Marne-La-Vallée \\ 5, Boulevard Descartes \\ 77455, Marne-La-Vallée Cedex, France \\ Email: capiez@univ-mlv.fr
}

\author{
Christian Soize \\ Laboratory of Engineering Mechanics (LaM) \\ University of Marne-La-Vallée \\ 5 , Boulevard Descartes \\ 77455 Marne-La-Vallée Cedex, France \\ Email: soize@univ-mlv.fr
}

\begin{abstract}
It is known that the forced response of mistuned bladed disks can strongly be amplified in comparison with the forced response of the tuned system. The random character of mistuning thus requires the construction of probabilistics models of random uncertainties. This paper presents a nonparametric probabilistic model of random uncertainties which is adapted to the problematics of the blade mistuning. This nonparametric approach allows all the uncertainties yielding mistuning (manufacturing tolerances, dispersion of materials) to be taken into account and includes also the uncertainties due to the modeling errors. This new probabilistic model takes into account both the mistuning of the blade eigenfrequencies and the blade modal shapes. The first point concerns the construction of this nonparametric approach in order to perform a mistuning analysis. The second part is devoted to the inverse problem associated with the manufacturing tolerances. A relationship between the manufacturing tolerances and the level of mistuning is also constructed.
\end{abstract}

\section{NOMENCLATURE \\ $N \quad$ Number of blades \\ $n_{a} \quad$ Number of DOFS of a blade \\ $n_{i} \quad$ Number of internal DOFS of a blade \\ $n_{\Sigma} \quad$ Number of DOFS on the coupling interface of a blade \\ $N_{g} \quad$ Number of generalized coordinates of a blade \\ $N_{a} \quad$ Dimension of the random reduced matrix of a blade \\ $N_{a}^{+} \quad$ Rank of the random reduced matrix of a blade \\ $\tilde{n} \quad$ Dimension of the second basis of modal reduction \\ $[A]^{T} \quad$ Transpose of matrix $[A]$}

\begin{tabular}{|c|c|}
\hline $\operatorname{tr}([A])$ & Trace of matrix $[A]$ \\
\hline $\operatorname{det}([A])$ & Determinant of square matrix $[A]$ \\
\hline$\|U\|$ & $\begin{array}{l}\text { Hermitian norm of vector } U \text { such that } \\
\|U\|=\left(U^{T} U\right)^{1 / 2}\end{array}$ \\
\hline$\|[A]\| \|_{F}$ & $\begin{array}{l}\text { Frobenius norm of matrix }[A] \text { such that } \\
\|[A]\|_{F}=\operatorname{tr}\left([A][A]^{T}\right)^{1 / 2}\end{array}$ \\
\hline$\left[I_{n}\right]$ & $n \times n$ identity matrix \\
\hline $\mathcal{E}$ & Mathematical expectation \\
\hline $\mathcal{P}(X>x)$ & $\begin{array}{l}\text { Probability that random variable } X \text { is greater } \\
\text { than a given level } x\end{array}$ \\
\hline $\mathbb{M}_{n, p}(\mathbb{R})$ & Set of all the $n \times p$ real matrices \\
\hline$M_{n}(\mathbb{R})$ & Set of all the $n \times n$ real matrices \\
\hline$M_{n}^{+}(\mathbb{R})$ & $\begin{array}{l}\text { Set of all the positive-definite symmetric } n \times n \\
\text { real matrices }\end{array}$ \\
\hline $\mathbb{M}_{n}^{+0}(\mathbb{R})$ & $\begin{array}{l}\text { Set of all the positive (semi-positive definite) } \\
\text { symmetric } n \times n \text { real matrices }\end{array}$ \\
\hline$\delta_{i j}$ & $\begin{array}{l}\text { Kronecker symbol such that } \delta_{i j}=1 \text { if } i=j \text { and } \\
\delta_{i j}=0 \text { if } i \neq j\end{array}$ \\
\hline $\mathbb{1}_{B}(b)$ & $\begin{array}{l}\text { Indicatrix function on any set } B \text { such that } \\
\mathbb{1}_{B}(b)=1 \text { if } b \in B \text { and } \mathbb{1}_{B}(b)=0 \text { if } b \ni B\end{array}$ \\
\hline
\end{tabular}

\section{INTRODUCTION}

In the context of turbomachinery rotors, it is fundamental to distinguish the nominal bladed-disk, which can be analyzed by using its cyclic symmetry [1] - [4] from the manufactured bladed-disk. As a matter of fact, manufacturing tolerances and dispersion of materials create many uncertainties on the geometry, on the boundary conditions and on the material properties of each blade. These uncertainties create small differences from 
one blade to another, which are referred as to mistuning. Unfortunately, the mistuning can induce strong vibrations for the forced response of the bladed-disks so that spatial localizations and large amplifications can be observed in the dynamic forced response on one or a few blades (see the early works [5] - [7]). The random character of the blade mistuning is a motivation to construct probabilistic models of random uncertainties. Parametric models are widely used to model the random uncertainties in structural dynamics. In the context of the blade mistuning, an exact parametric approach would require to identify all the uncertain parameters which cause mistuning. Furthermore, a complete probabilistic parametric description should be required, including the use of stochastic fields for modeling geometric uncertainties. Then functions mapping the domain of uncertain parameters into the mass, damping and stiffness finite element matrices should be constructed. Such a probabilistic parametric method is not very simple to introduce in the context of the mistuned bladed-disks and above all requires a big experimental effort in order to estimate all the probabilistic quantities allowing stochastic fields to be decribed using mathematical statistics. The very simple probabilistic model of random uncertainties which consist in modeling the mistuning by considering Young modulus uncertainties of the blades seems to be very unsufficient. Blade eigenfrequencies are mistuned through their stiffnesses parameters but the corresponding eigenmodes remain unchanged with respect to the tuned case (see for instance [8] - [12] for lumped parameters models, [13] - [15] for reduced order models issued from finite element models). Recently, probabilistic approaches concerning the blade eigenmodes mistuning have been developed [16] - [19] and showed that there exist differences with respect to the probabilistic approach currently used. In this paper, the construction of a new probabilistic model of random uncertainties referred as to a nonparametric approach is presented. Such a nonparametric approach differs from a parametric one because the knowledge of the uncertain parameters is not required. The probability model is directly constructed from the generalized matrices which result from a mean reduced matrix model. It consists of a probability model for symmetric positive-definite real random matrices which has been constructed using the maximum entropy principle and using the available information [20] - [21]. The available information includes a mean reduced matrix model which is constructed by dynamic substructuring of the bladed-disk with cyclic symmetry. This nonparametric probabilistic model takes into account all uncertainties yielding mistuning, that is to say, it includes blade eigenfrequencies and blade modal shapes uncertainties on a coherent way. The first point of this paper deals with the construction of this nonparametric approach in order to perform a mistuning analysis.

Furthermore, one of the major cause concerning the blade mistuning is mainly due to the tolerances encountered during the manufacturing process of the blade (outline shapes, surfaces finishing...). The tolerances specified during the conception phase define the acceptable margin of fluctuations of the blade characteristics allowing the bladed-disk to work correctly. In a context of mistuning, the good working of a turbomachinery is charac- terized by the amplification factor between the mistuned and the maximum tuned response over a frequency band. In the industry of turbomachineries, a leading stake is to specify the tolerancing rate for which the amplification of response does not exceed a critical magnification factor [22]. Such an inverse problem using a probabilistic parametric approach would not be appropriate due to the complexity of modeling tolerances. An original methodology is proposed for solving this inverse problem using the nonparametric approach. The robustness of such a probabilistic model is justified by its simplicity since it requires the only use of a reduced matrix model of a blade and since each random matrix (mass, stiffness and damping) is characterized by one scalar allowing the dispersion of the model to be controlled.

In a first part, the mean reduced matrix model for a blade is constructed; the following part is devoted to the construction of the random reduced matrix model using the main results concerning the nonparametric model of random uncertainties. The third part presents the methodology of the mistuning analysis for the complete structure. In a fourth part, the construction of an a priori probabilistic model for manufacturing tolerances is formulated. This probabilistic model is constructed in the goal to identify the data parameters used by the probability model of the nonparametric approach. The methodology for solving the inverse problem for specifying manufacturing tolerances is then investigated. Finally, a numerical example is presented.

\section{MEAN REDUCED MATRIX MODEL FOR A BLADE}

We are interested in the linearized vibrations of a fixed structure with an $\mathrm{N}$-order cyclic symmetry around a static equilibrium configuration considered as a natural state without prestresses and whose model will be called the mean model of the structure. The structure is submitted to external forces and the vibration analysis is performed in the frequency band $\mathbb{B}$. The methodology and the results presented can be extended without difficulty to the case of rotating structures. The structure $\Omega$ is divided into $N+1$ substructures, that is to say one disk $\Omega_{d}$ fixed on a part of his boundary and $N$ identical blades $\Omega_{j}$, for $j$ belonging to $\{0, \ldots, N-1\}$ with free boundary. Each blade $j$ is coupled with the disk by the coupling interface $\Sigma_{j}$ such that $\bigcup_{j \in\{0, \ldots, N-1\}} \Sigma_{j}=\Sigma$. Since the mistuning is assumed to be statistically independent from blade to blade, the blades are treated as individual components which are reduced by the Craig \& Bampton method [23] while the disk remains a nonreduced substructure. In order to simplify the mathematical notations, we choose to cancel the exponent $j$ related to blade $j$ when no confusion is possible.

For all $\omega$ in band $\mathbb{B}$, the mean finite element model for blade $j$ with free coupling interface is written as

$$
\left(-\omega^{2}[\underline{M}]+i \omega[\underline{D}]+[\underline{K}]\right) \underline{\mathbf{u}}(\omega)=\underline{\mathbf{f}}(\omega)
$$

in which $\underline{\mathbf{u}}(\omega)$ and $\underline{\mathbf{f}}(\omega)$ are the $\mathbb{C}^{n_{a}}$-valued vectors of the mean displacements and of the external forces respectively and where 
$[\underline{M}],[\underline{D}]$ and $[\underline{K}]$ are the mean mass, damping and stiffness symmetric $n_{a} \times n_{a}$ real matrices. The mass matrix belongs to $\mathbb{M}_{n_{a}}^{+}(\mathbb{R})$ while damping and stiffness matrices belong to $M_{n_{a}}^{+0}(\mathbb{R})$ since $\Omega_{j}$ has a free boundary. The block decomposition with respect to the $n_{i}$ internal and $n_{\Sigma}=n_{a}-n_{i}$ coupling interface DOFs is then written as

$$
\begin{gathered}
\underline{\mathbf{u}}(\omega)=\left[\begin{array}{c}
\underline{\mathbf{u}}_{i}(\omega) \\
\underline{\mathbf{u}}_{\Sigma}(\omega)
\end{array}\right], \quad \underline{\mathbf{f}}(\omega)=\left[\begin{array}{c}
\underline{\mathbf{f}}_{i}(\omega) \\
\underline{\mathbf{f}}_{\Sigma}(\omega)
\end{array}\right]+\left[\begin{array}{c}
0 \\
\underline{\mathbf{f}}_{\Sigma, c}(\omega)
\end{array}\right], \\
{[\underline{E}]=\left[\begin{array}{cc}
{\left[\underline{E}_{i i}\right]} & {\left[\underline{E}_{i \Sigma}\right]} \\
{\left[\underline{E}_{i \Sigma}\right]^{T}} & {\left[\underline{E}_{\Sigma \Sigma}\right]}
\end{array}\right] \quad, \quad E=\{M, D, K\} .}
\end{gathered}
$$

The Craig \& Bampton decomposition [23] with $N_{g}$ fixed coupling interface modes is written as

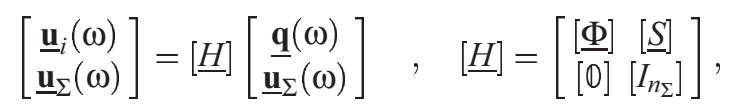

where $\underline{\mathbf{q}}(\omega)$ is the $\mathbb{C}^{N_{g}}$-valued vector of the mean generalized coordinates of blade $j,[\underline{\Phi}]$ is the $\mathbb{M}_{n_{i}, N_{g}}(\mathbb{R})$-valued matrix whose columns are the eigenvectors $\left\{\underline{\varphi}_{1}, \underline{\varphi}_{2}, \ldots, \underline{\varphi}_{N_{g}}\right\}$ of the structure with a fixed coupling interface, $[\underline{S}]$ is the $\mathbb{M}_{n_{i}, n_{\Sigma}}(\mathbb{R})$-valued matrix defined by $[\underline{S}]=-\left[\underline{K}_{i i}\right]^{-1}\left[\underline{K}_{i \Sigma}\right]$. Using Eqs (1),(4) we obtain

$$
\left[\underline{A}_{\mathrm{red}}(\omega)\right]\left[\begin{array}{c}
\underline{\mathbf{q}}(\omega) \\
\underline{\mathbf{u}}_{\Sigma}(\omega)
\end{array}\right]=\left[\begin{array}{c}
{[\underline{\Phi}]^{T} \underline{\mathbf{f}}_{i}(\omega)} \\
{[\underline{S}]^{T} \underline{\mathbf{f}}_{i}(\omega)+\underline{\mathbf{f}}_{\Sigma}(\omega)+\underline{\mathbf{f}}_{\Sigma, c}(\omega)}
\end{array}\right]
$$

where $\left[\underline{A}_{\text {red }}(\omega)\right]$ defines the mean reduced dynamic stiffness of the blade such that

$$
\left[\underline{A}_{\mathrm{red}}(\omega)\right]=-\omega^{2}\left[\underline{M}_{\mathrm{red}}\right]+i \omega\left[\underline{D}_{\mathrm{red}}\right]+\left[\underline{K}_{\mathrm{red}}\right]
$$

and where $\left[\underline{M}_{\text {red }}\right],\left[\underline{D}_{\text {red }}\right],\left[\underline{K}_{\text {red }}\right]$ are such that, defining $N_{a}$ as $N_{a}=$ $N_{g}+n_{\Sigma}$

$$
\begin{aligned}
& {\left[\underline{M}_{\text {red }}\right]=[\underline{H}]^{T}[\underline{M}][\underline{H}],\left[\underline{M}_{\text {red }}\right] \in \mathbb{M}_{N_{a}}^{+}(\mathbb{R}),} \\
& {\left[\underline{D}_{\text {red }}\right]=[\underline{H}]^{T}[\underline{D}][\underline{H}],\left[\underline{D}_{\text {red }}\right] \in M_{N_{a}}^{+0}(\mathbb{R}),} \\
& {\left[\underline{K}_{\text {red }}\right]=[\underline{H}]^{T}[\underline{K}][\underline{H}],\left[\underline{K}_{\text {red }}\right] \in \mathbb{M}_{N_{a}}^{+0}(\mathbb{R}) .}
\end{aligned}
$$

Introducing the block decomposition derived from Eq. (5), we obtain

$$
\left[\underline{A}_{\mathrm{red}}(\omega)\right]=\left[\begin{array}{cc}
{\left[\underline{\mathcal{A}}^{\mathcal{A}}(\omega)\right]} & {\left[\underline{A}_{c}(\omega)\right]} \\
{\left[\underline{A}_{c}(\omega)\right]^{T}} & {\left[\underline{A}_{\Sigma}(\omega)\right]}
\end{array}\right]
$$

\section{NONPARAMETRIC MODEL OF RANDOM UNCERTAIN- TIES FOR BLADES MISTUNING}

The nonparametric approach is based on the use of a probabilistic model constructed from the generalized matrices resulting from a mean reduced matrix model. The probability model is constructed on the set of all the positive-definite matrices using the available information and the maximum entropy principle. The available information concerns the knowledge of the mean reduced matrices which are equal to the mean values of the random reduced matrices and concerns the existence of the second-order moment of their inverse. The last condition assure the existence of the random response $\{\mathbf{U}(\omega), \omega \in \mathbb{B}\}$ as a secondorder stochastic process indexed by $\mathbb{B}$. The basis of this theory is completely developed and validated for linear [20,21] and for nonlinear [24] problems. In particular, the construction of the probability model and the necessity of normalizing the random matrices are justified. Furthermore, substructuring techniques allowing nonhomogeneous uncertainties to be described and using the nonparametric approach have been validated [25].

\section{Random reduced matrix model for the blade}

First of all, the bladed-disk is decomposed in substructures in order to consider random uncertainties which are statistically independent from blade to blade. Thus the following reasoning can be limited to one blade $j$. The main results concerning the nonparametric model of random uncertainties in structural dynamics are used (see [20], [21], [24] and [25]) and adapted to the mistuning problematics of each blade. Such a probabilistic model is not implemented from a mean finite element matrix model of the blade but from a mean reduced matrix model of the blade. Since the investigated structure is the whole bladeddisk, the mean reduced matrix model of the blade has to allow a reduced matrix model of the whole structure to be constructed. This justifies the use of the Craig and Bampton dynamic substructuring method, which keeps the degrees of freedom of the coupling interface. Thus, the probabilistic nonparametric model of the blade is implemented from the mean reduced matrix model described above. The random vibration equation derived from Eq. (5) is then written as

$$
\left[\mathbf{A}_{\text {red }}(\omega)\right]\left[\begin{array}{c}
\mathbf{Q}(\omega) \\
\mathbf{U}_{\Sigma}(\omega)
\end{array}\right]=\left[\begin{array}{c}
{[\underline{\Phi}]^{T} \underline{\mathbf{f}}_{i}(\omega)} \\
{[\underline{S}]^{T} \underline{\mathbf{f}}_{i}(\omega)+\underline{\mathbf{f}}_{\Sigma}(\omega)+\mathbf{F}_{\Sigma, c}(\omega)}
\end{array}\right]
$$

where

$$
\left[\mathbf{A}_{\text {red }}(\omega)\right]=-\omega^{2}\left[\mathbf{M}_{\mathrm{red}}\right]+i \omega\left[\mathbf{D}_{\mathrm{red}}\right]+\left[\mathbf{K}_{\mathrm{red}}\right]
$$

is the random dynamic stiffness of the free-interfaced blade $j$ in which $\left[\mathbf{M}_{\text {red }}\right],\left[\mathbf{D}_{\text {red }}\right]$ and $\left[\mathbf{K}_{\text {red }}\right]$ are respectively $\mathbf{M}_{N_{a}}^{+}(\mathbb{R})$-, $M_{N_{a}}^{+0}(\mathbb{R})$ - and $M_{N_{a}}^{+0}(\mathbb{R})$-valued independent random variables corresponding to the random reduced mass, damping and stiffness matrices and such that

$$
\mathcal{E}\left\{\left[\mathbf{M}_{\text {red }}\right]\right\}=\left[\underline{M}_{\text {red }}\right],
$$




$$
\begin{aligned}
& \mathcal{E}\left\{\left[\mathbf{D}_{\text {red }}\right]\right\}=\left[\underline{D}_{\text {red }}\right], \\
& \mathcal{E}\left\{\left[\mathbf{K}_{\text {red }}\right]\right\}=\left[\underline{K}_{\text {red }}\right] .
\end{aligned}
$$

The vectors $\mathbf{F}_{\Sigma, c}(\omega), \mathbf{U}_{\Sigma}(\omega)$ and $\mathbf{Q}(\omega)$ define the random coupling forces, the random displacements acting on coupling interface $\Sigma_{j}$ and the random generalized coordinates, respectively. The dispersion level of these three independent random matrices are then controlled by the three scalar parameters $\delta_{M}, \delta_{D}, \delta_{K}$ which are called the dispersion parameters.

\section{Probability model of the random matrices for one blade}

In order to construct the probability model of the random independent matrices [ $\left.\mathbf{M}_{\mathrm{red}}\right]$, [ $\left.\mathbf{D}_{\mathrm{red}}\right]$ and $\left[\mathbf{K}_{\mathrm{red}}\right]$, a normalization process has to be done before constructing the probability model.

Normalization of the random reduced matrices The normalization of random reduced matrices $\left[\mathbf{M}_{\text {red }}\right]$, $\left[\mathbf{D}_{\text {red }}\right]$ and $\left[\mathbf{K}_{\text {red }}\right]$ requires the factorization of their mean matrices. Since mean mass matrix $\left[\underline{M}_{\text {red }}\right]$ is positive-definite, the Cholesky factorization yields

$$
\left[\underline{M}_{\mathrm{red}}\right]=\left[\underline{L}_{M}\right]^{T}\left[\underline{L}_{M}\right],
$$

in which $\left[\underline{L}_{M}\right]$ is an upper triangular matrix in $\mathbb{M}_{N_{a}}(\mathbb{R})$. Since matrices $\left[\underline{K}_{\text {red }}\right]$ and $\left[\underline{D}_{\text {red }}\right]$ are positive semi-definite with a rank assumed to be equal to $N_{a}^{+}$, the factorization is carried out thanks to the spectral decomposition of the matrices. It can easily be proved that

$$
\left[\underline{K}_{\mathrm{red}}\right]=\left[\underline{L}_{K}\right]^{T}\left[\underline{L}_{K}\right] \quad, \quad\left[\underline{D}_{\mathrm{red}}\right]=\left[\underline{L}_{D}\right]^{T}\left[\underline{L}_{D}\right]
$$

where $\left[\underline{L}_{K}\right]$ and $\left[\underline{L}_{D}\right]$ are belonging to $\mathbb{M}_{N_{a}^{+}, N_{a}}(\mathbb{R})$. Each random matrix is then written as

$$
\begin{aligned}
{\left[\mathbf{M}_{\mathrm{red}}\right] } & =\left[\underline{L}_{M}\right]^{T}\left[\mathbf{G}_{M}\right]\left[\underline{L}_{M}\right], \\
{\left[\mathbf{D}_{\mathrm{red}}\right] } & =\left[\underline{L}_{D}\right]^{T}\left[\mathbf{G}_{D}\right]\left[\underline{L}_{D}\right], \\
{\left[\mathbf{K}_{\mathrm{red}}\right] } & =\left[\underline{L}_{K}\right]^{T}\left[\mathbf{G}_{K}\right]\left[\underline{L}_{K}\right],
\end{aligned}
$$

in which $\left[\mathbf{G}_{M}\right],\left[\mathbf{G}_{D}\right]$ and $\left[\mathbf{G}_{K}\right]$ are independent normalized random matrices with values in $\mathbb{M}_{N_{a}}^{+}(\mathbb{R}), \mathbb{M}_{N_{a}^{+}}^{+}(\mathbb{R})$ and $M_{N_{a}^{+}}^{+}(\mathbb{R})$.

\section{Construction of the probability model of the} normalized random matrices

From Eqs. (13),(14),(15),(18),(19) and (20), it can be deduced that the mean values of random matrices $\left[\mathbf{G}_{M}\right],\left[\mathbf{G}_{D}\right]$ and $\left[\mathbf{G}_{K}\right]$ are such that

$$
\begin{aligned}
& \mathcal{E}\left\{\left[\mathbf{G}_{M}\right]\right\}=\left[\underline{G}_{M}\right]=\left[I_{N_{a}}\right], \\
& \mathcal{E}\left\{\left[\mathbf{G}_{D}\right]\right\}=\left[\underline{G}_{D}\right]=\left[I_{N_{a}^{+}}\right], \\
& \mathcal{E}\left\{\left[\mathbf{G}_{K}\right]\right\}=\left[\underline{G}_{K}\right]=\left[I_{N_{a}^{+}}\right] .
\end{aligned}
$$

The dispersion of these random matrices are controlled by the strictly positive real parameters $\delta_{M}, \delta_{D}$ and $\delta_{K}$ defined by

$$
\delta_{E}=\left\{\frac{\mathcal{E}\left\{\left\|\left[\mathbf{G}_{E}\right]-\left[\underline{G}_{E}\right]\right\|_{F}^{2}\right\}}{\left\|\left[\underline{G}_{E}\right]\right\|_{F}^{2}}\right\}^{\frac{1}{2}} \text { with } \quad E=\{M, D, K\} .
$$

Below, $[\mathbf{G}]$ denotes $\left[\mathbf{G}_{M}\right],\left[\mathbf{G}_{D}\right]$ and $\left[\mathbf{G}_{K}\right], n$ denotes $N_{a}, N_{a}^{+}$and $N_{a}^{+}$and $\delta$ denotes $\delta_{M}, \delta_{D}$ and $\delta_{K}$. From the theory [20,21], it is proved that for fixed $n \geq n_{0}$, where $n_{0}$ is a fixed integer such that $n_{0} \geq 1$, dispersion parameter $\delta$ has to be chosen such that

$$
0<\delta<\sqrt{\frac{n_{0}+1}{n_{0}+5}}
$$

It should be noted that, in practice, $n_{0}$ is chosen equal to $n$. Since in general, the rank of the random matrix is large enough, the upper bound of parameter $\delta$ is close to 1 . Then the probability density function with respect to the volume element

$$
\tilde{d} G=2^{n(n-1) / 4} \prod_{1 \leq i \leq j \leq n} d[G]_{i j}
$$

is written as

$$
\begin{aligned}
& p_{[\mathbf{G}]}([G])=\mathbb{1}_{\mathbb{M}_{n}^{+}(\mathbb{R})}([G]) \times C_{G} \\
& \quad \times(\operatorname{det}([G]))^{\left(1-\delta^{2}\right)(2 \delta)^{-1}(n+1)} \times e^{-(n+1)(2 \delta)^{-1} \operatorname{tr}([G])},
\end{aligned}
$$

where $C_{G}$ is the positive constant of normalization such that

$$
C_{G}=\frac{(2 \pi)^{-n(n-1) / 4}\left(\frac{n+1}{2 \delta^{2}}\right)^{n(n+1)\left(2 \delta^{2}\right)^{-1}}}{\prod_{j=1}^{n} \Gamma\left(\frac{n+1}{2 \delta^{2}}+\frac{1-j}{2}\right)},
$$

in which $\Gamma(z)$ is the gamma function defined for all $z>0$ as $\Gamma(z)=\int_{0}^{\infty} t^{z-1} e^{-t} d t$.

The covariance tensor defined by

$$
C_{j k, j^{\prime} k^{\prime}}^{G}=\mathcal{E}\left\{\left([\mathbf{G}]_{j k}-[\underline{G}]_{j k}\right)\left([\mathbf{G}]_{j^{\prime} k^{\prime}}-[\underline{G}]_{j^{\prime} k^{\prime}}\right)\right\}
$$

is such that

$$
C_{j k, j^{\prime} k^{\prime}}^{G}=\frac{\delta^{2}}{n+1}\left\{\delta_{j^{\prime} k} \delta_{j k^{\prime}}+\delta_{j j^{\prime}} \delta_{k k^{\prime}}\right\}
$$

Algebraic representation of normalized matrices The following algebraic representation of random positive-definite symmetric real matrix $[\mathbf{G}]$ allows a procedure for the Monte 
Carlo numerical simulation of random matrix $[\mathbf{G}]$ to be defined. Random matrix $[\mathbf{G}]$ is written as

$$
[\mathbf{G}]=\left[\mathbf{L}_{G}\right]^{T}\left[\mathbf{L}_{G}\right]
$$

in which $\left[\mathbf{L}_{G}\right]$ is an upper triangular random matrix with values in $M_{n}(\mathbb{R})$ such that

(1) random variables $\left\{\left[\mathbf{L}_{G}\right]_{j j^{\prime}}, j \leq j^{\prime}\right\}$ are independent;

(2) for $j<j^{\prime}$, real-valued random variable $\left[\mathbf{L}_{G}\right]_{j j^{\prime}}$ can be written as $\left[\mathbf{L}_{G}\right]_{j j^{\prime}}=\sigma_{n} U_{j j^{\prime}}$ in which $\sigma_{n}=\delta(n+1)^{-1 / 2}$ and where $U_{j j^{\prime}}$ is a real-valued Gaussian random variable with zero mean and variance equal to 1 ;

(3) for $j=j^{\prime}$, positive-valued random variable $\left[\mathbf{L}_{G}\right]_{j j}$ can be written as $\left[\mathbf{L}_{G}\right]_{j j}=\sigma_{n} \sqrt{2 V_{j}}$ in which $\sigma_{n}$ is defined above and where $V_{j}$ is a positive-valued gamma random variable whose probability density function $p_{V_{j}}(v)$ with respect to $d v$ is written as

$$
p_{V_{j}}(v)=\mathbb{1}_{\mathbb{R}^{+}}(v) \frac{1}{\Gamma\left(\frac{n+1}{2 \delta^{2}}+\frac{1-j}{2}\right)} v^{\frac{n+1}{2 \delta^{2}}-\frac{1+j}{2}} e^{-v} .
$$

\section{METHODOLOGY FOR MISTUNING ANALYSIS}

The mean reduced matrix model for one blade is constructed and is assembled with the mean finite element model of the disk sector. Then, cyclic symmetry is used to compute the mean eigenvectors related to the mean generalized eigenvalue problem of the mean reduced matrix model for the bladed disk. The mean eigenvectors associated with the $\tilde{n}$ first mean eigenvalues constitute the mean projection basis which will be used to reduce the cost of the numerical simulations. The mistuning analysis is performed using a Monte Carlo numerical simulation. Each realization of the random mass, damping and stiffness matrices related to the random reduced matrix model of each blade is constructed using the algebraic representation described above. The assemblage of these random matrices related to the blades with the mean finite element model of the disk is carried out and the random reduced matrix model of the complete structure is reduced on the mean projection basis. A realization of the random observation is then computed. Such a random observation is the random dynamical magnification factor $\{\mathbf{B}(\omega), \omega \in \mathbb{B}\}$, which is the positive real-valued stochastic process indexed by $\mathbb{B}$ such that

$$
\mathbf{B}(\omega)=\sup _{j \in\{0, \ldots, N-1\}} \frac{\left\|\mathbf{U}^{j}(\omega)\right\|}{\left\|\underline{\mathbf{u}}^{j}\right\|_{\infty}},\left\|\underline{\mathbf{u}}^{j}\right\|_{\infty}=\sup _{\omega \in \mathbb{B}}\left\|\underline{\mathbf{u}}^{j}(\omega)\right\|,
$$

where $\mathbf{U}^{j}(\omega)$ is the displacement of the mistuned blade $j$ and where $\underline{\mathbf{u}}^{j}(\omega)$ is the displacement of the tuned blade $j$. Moreover, the random dynamical magnification factor over narrow frequency band $\mathbb{B}$ is defined by

$$
B_{\infty}=\sup _{\omega \in \mathbb{B}} \mathbf{B}(\omega)
$$

The $n_{s}$ realizations $B_{\infty}\left(\theta_{1}\right), \ldots, B_{\infty}\left(\theta_{n_{s}}\right)$ of random observation $B_{\infty}$ are then calculated and mathematical statistics are used for estimating probabilistic quantities related to random variable $B_{\infty}$.

\section{PROBABILISTIC MODEL FOR MANUFACTURING TOL- ERANCES}

In order to estimate the dispersion parameters $\delta_{M}$ and $\delta_{K}$ of the nonparametric model related to the blades, a parametric model of random geometrical uncertainties of the blades is introduced. The construction of this a priori model is done in the goal to establish a relationship between the tolerance specification and the dispersion parameters $\delta_{M}$ and $\delta_{K}$ of the nonparametric approach. In other terms, if the parametric probabilistic model was used in the way to model the geometrical uncertainties, the resulting mistuning analysis would be wrong due to the relationship between the uncertain parameters and the displacement field which is nonlinear. It should be noted that the nonparametric probabilistic model uses a probability distribution which is completely coherent with the available information. Then, the parametric probabilistic model presented in this Section is not used for constructing the probability density function of the nonparametric model but is only used for estimating the value of the dispersion parameters $\delta_{M}$ and $\delta_{K}$ which are the free parameters of the nonparametric model. From now on, it is assumed that all the random parametric uncertainties inducing the mistuning of the blade are mainly due to the geometrical perturbations of the blades. Since we are interested in the mistuning induced by the manufacturing process of the blades, then the analysis can be carried out for one blade.

\section{Construction of an a priori parametric probabilistic model}

Inaccuracies are created during the manufacturing process. Consequently, all the manufactured blades are different from each other and from the nominal blade. This nominal blade is used to specify the tolerances, which are the authorized margin of the blade-characteristics fluctuations. The dimensional tolerances are defined as the upper bound that the dimensions can reach with respect to the nominal dimension (chord or length of the blade...). The geometrical tolerances are related to the variations of the global form with respect to the nominal blade form (authorized spherical envelop centered on the nominal blade profile...). The principle of independency precises that each dimensional or geometrical requirement should occur independently. Surface tolerances of higher order like waves or roughness can be defined too. Let $s \in \mathbb{N}^{*}$ and let $\underline{t} \in \mathbb{R}^{s}$ be the vector of the nominal parameters from which the tolerances can be defined. A probabilistic model of the fluctuations around these parameters has to be defined. Let $\mathbf{T}=\left(T_{1}, \ldots, T_{S}\right)$ be a second-order random variable with values in $B^{s} \in \mathbb{R}^{s}$ characterizing the fluctuation of all the parameters involved by tolerancing. Let $B^{S}$ be 
such that:

$$
B^{s}=\prod_{r=1}^{s}\left[-\tau_{r}, \tau_{r}\right] \quad, \quad \tau_{r} \in \mathbb{R}^{+} \quad, \quad \forall r \in\{1 \ldots s\}
$$

where $\tau_{r}$ or $-\tau_{r}$ define the upper or lower allowable fluctuation of parameter $\mathbf{T}_{r}$. Using the independency principle, random variables $T_{1}, \ldots, T_{s}$ are independent. Consequently, probability density function $p_{\mathbf{T}}(t)$ with respect to measure $d t=d t_{1} \ldots d t_{s}$ of $\mathbb{R}^{s}$ is written as

$$
p_{\mathbf{T}}(t)=p_{T_{1}, \ldots, T_{s}}\left(t_{1}, \ldots, t_{s}\right)=\prod_{r=1}^{s} p_{T_{r}}\left(t_{r}\right) .
$$

Using Eq. (35) and using the maximum entropy principle, the probability density function of random variable $\mathbf{T}$ is written as

$$
p_{\mathbf{T}}(t)=\prod_{r=1}^{s} \frac{1}{2 \tau_{r}}
$$

Consequently, $\forall r \in\{1, \ldots, s\}, T_{r}$ is a centered uniform random variable whose standard deviation is such that $\sigma_{r}=\tau_{r} / \sqrt{3}$.

\section{Finite element discretization}

Let $\Omega$ be the domain of the nominal blade and let $\tilde{\Omega}(\mathbf{T})$ be the random domain of the manufactured blade. These domains are such that there exists a one-to-one mapping $g$ defined by

$$
\begin{aligned}
\Omega & \longrightarrow \tilde{\Omega}(\mathbf{T}) \\
x & \mapsto g(x ; \mathbf{T}),
\end{aligned}
$$

such that $\forall x \in \Omega, \tilde{x}=g(x ; \mathbf{T})$ with $\operatorname{det} \frac{\partial g(x ; \mathbf{T})}{\partial x}>0$ almost surely. Mass density $\tilde{\rho}$ and elasticity tensor $\tilde{a}$ are defined on $\tilde{\Omega}(\mathbf{T})$ by

$$
\begin{aligned}
& \tilde{\rho}(\tilde{x})=\rho(x), \\
& \tilde{\mathbb{a}}(\tilde{x})=\mathbb{a}(x), \quad \tilde{x}=g(x ; \mathbf{T}) \quad, \quad \forall x \in \Omega,
\end{aligned}
$$

where $\rho$ and $₫$ are the mass density and the elasticity tensor of the nominal blade. Let $m_{j}, j \in\left\{1, \ldots, N_{n}\right\}$ and $M_{j}, j \in\left\{1, \ldots, N_{n}\right\}$ be the nodes of the mesh of $\Omega$ and $\tilde{\Omega}(\mathbf{T})$, where $N_{n}$ denote the number of nodes of the mesh. The nodes $m_{1}, \ldots, m_{p}$ and $M_{1}, \ldots, M_{p}$, $p<N_{n}$ are the nodes belonging to $\partial \Omega$ and $\partial \Omega(\mathbf{T})$ respectively. $M_{j}$ is explicitly defined by

$$
\begin{aligned}
M_{j}=f_{j}\left(m_{1}, \ldots, m_{N_{n}} ; \mathbf{T}\right) \quad & , \quad \forall j \in\{1, \ldots, p\}, \\
M_{j}=m_{j} & , \quad \forall j \in\left\{p+1, \ldots, N_{n}\right\} .
\end{aligned}
$$

The mappings $f_{1}, \ldots, f_{p}$ can easily be constructed from the parameterized surface. Let $\{[M(\mathbf{T})],[K(\mathbf{T})]\}$ be the random mass and stiffness matrices with values in $\left\{M_{n_{a}}^{+}(\mathbb{R}) \times M_{n_{a}}^{+0}(\mathbb{R})\right\}$ resulting from the finite element discretization of $\tilde{\Omega}(\mathbf{T})$.

\section{METHODOLOGY FOR THE INVERSE PROBLEM USING THE NONPARAMETRIC APPROACH}

In the previous section, a probabilistic parametric model of random uncertainties has been constructed to model the manufactured blade using the parameters which characterize the manufacturing tolerances. Nevertheless, its construction remains arbitrary so that it does not constitute an exact modeling of the problem. Indeed, an exact parametric approach would require to identify all the uncertain parameters and to construct the probability distribution of stochastic fields. However, this model provides a global information which allow a relationship between the upper bounds $\tau_{1}, \ldots, \tau_{s}$ and the dispersion parameters $\delta_{M}, \delta_{K}$ related to the nonparametric probabilistic model of random uncertainties to be constructed. Once the dispersion parameters are determined, a mistuning analysis is performed using the nonparametric approach. Such an approach is theoretically exact because all the uncertain parameters are taken into account, because algebraic and mathematical properties are conserved (existence of the second-order moments of the random response, ...), and because only the mean reduced model of the blade is required. It should be noted that the nonparametric model of random uncertainties includes the errors due to the choice of the model since the uncertainties of the continuous structure which cannot be seen by the model are considered through this approach.

\section{Identifying dispersion parameters $\delta_{M}$ and $\delta_{K}$ with re- spect to the upper bounds of the tolerances}

In order to calculate dispersion parameters $\delta_{M}$ and $\delta_{K}$, a criterion has to be constructed in order to establish a global relationship between the two probabilistic models. The purpose of such a criterion is to characterize the dispersion parameters $\delta_{M}$ and $\delta_{K}$ of the nonparametric model, which correspond to a given value of the upper bound vector $\tau=\left(\tau_{1}, \ldots, \tau_{s}\right)$. Since the nonparametric probabilistic model of a blade is constructed from a mean reduced matrix model related to the nominal blade, this criterion has necessarily to be defined in the mean basis of the reduced coordinates related to the nominal blade corresponding to the mean reduced matrix model. Furthermore, the manufactured blade characteristics have to be compared with their nominal characteristics. Thus, the fluctuations of the random matrices around their nominal value are studied in the common basis of projection related to the nominal blade for each probabilistic model. Below, $B \in\{M, K\}$. The random matrix $[B(\mathbf{T})]$ is first projected using the mean basis,

$$
\left[B_{\text {red }}(\mathbf{T})\right]=[\underline{H}]^{T}[B(\mathbf{T})][\underline{H}]
$$

where $\left[B_{\text {red }}(\mathbf{T})\right]$ is the $\mathbb{M}_{N_{a}}^{+}(\mathbb{R})$ (or $\left.\mathbb{M}_{N_{a}}^{+0}(\mathbb{R})\right)$-valued random reduced mass or stiffness matrix. Let $W_{B}^{\text {tol }}(\boldsymbol{\tau})$ be the scalar parameter characterizing the dispersion of matrix $\left[B_{\text {red }}(\mathbf{T})\right]$ with respect to $\left[\underline{B}_{\text {red }}\right]$, which is defined by

$$
W_{B}^{\mathrm{tol}}(\boldsymbol{\tau})=\mathcal{E}\left\{\left\|\left[B_{\text {red }}(\mathbf{T})\right]-\left[\underline{B}_{\text {red }}\right]\right\|_{F}^{2}\right\},
$$


and which depends only on the upper bound vector $\tau$. For the nonparametric model, the similar parameter $W_{B}$ is defined by

$$
W_{B}=\mathcal{E}\left\{\|\left[\mathbf{B}_{\text {red }}\right]-\left.\left[\underline{B}_{\text {red }}\right]\right|_{F} ^{2}\right\} .
$$

The tolerances are described through the nonparametric approach by identifying scalar parameters $W_{B}^{\text {tol }}(\boldsymbol{\tau})$ and $W_{B}$ such that

$$
W_{B}^{\mathrm{tol}}(\boldsymbol{\tau})=W_{B}
$$

Using Eqs. (18) to (20),(29), (42) to (44) and the symmetry properties of the random matrices yields

$$
\begin{aligned}
& \delta_{K}(\boldsymbol{\tau})=\sqrt{\frac{W_{K}^{\mathrm{tol}}(\boldsymbol{\tau})\left(N_{a}^{+}+1\right)}{\operatorname{tr}\left(\left[\underline{K}_{\mathrm{red}}\right]\right)^{2}+\operatorname{tr}\left(\left[\underline{K}_{\mathrm{red}}\right]^{2}\right)}}, \\
& \delta_{M}(\boldsymbol{\tau})=\sqrt{\frac{W_{M}^{\mathrm{tol}}(\boldsymbol{\tau})\left(N_{a}+1\right)}{\operatorname{tr}\left(\left[\underline{M}_{\mathrm{red}}\right]\right)^{2}+\operatorname{tr}\left(\left[\underline{M}_{\mathrm{red}}\right]^{2}\right)}} .
\end{aligned}
$$

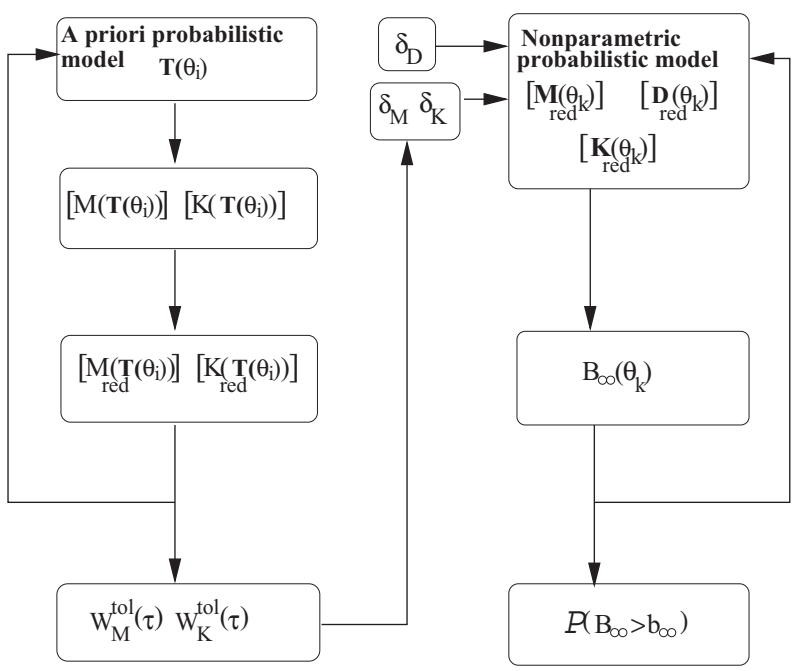

Figure 1. Procedure for solving the inverse problem

\section{Solving the inverse problem}

The inverse approach is solved by using two Monte Carlo numerical simulations, as described in Fig. 1. The procedure is constructed as follows: a first Monte Carlo numerical simulation is performed on one blade. For each realization, the finite-element mass and stiffnesses matrices of the blade are constructed by using the probabilistic model on the mesh derived from the parametric probabilistic model related to the tolerances characteristics. Once these matrices are reduced on the mean basis, the relationship between tolerances upper bound vector $\tau$ and dispersion parameters $\delta_{M}$ and $\delta_{K}$ of the nonparametric model is estimated. Then, dispersion parameter $\delta_{D}$ is introduced independently. A second Monte Carlo numerical simulation is performed using the mistuning analysis procedure described above. In the context of manufacturing tolerances, $\mathcal{P}\left(B_{\infty}>b_{\infty}\right)$ is the quantity which has to be estimated, in which $b_{\infty}$ is the critical dynamical magnification factor. Performing a parametric study with respect to the upper bound vector $\tau$ allows the inverse problem to be solved. For a given critical dynamical magnification factor $b_{\infty}$ and for a given probability level $\mathcal{P}\left(B_{\infty}>b_{\infty}\right)$, the required manufacturing tolerances can be specified. It should be noted that the numerical simulations related to the nonparametric approach are directly performed from the reduced matrix model of each blade. With this approach the first Monte Carlo procedure is applied to the finite element model of one blade. The second Monte Carlo procedure is applied to $N$ mean reduced matrix models of a blade. It should be noted that the case of a parametric approach would require only one Monte Carlo numerical procedure but would need $N$ finite elements models of a blade per sample. Consequently, with the nonparametric approach, the computational savings with regard to an exact parametric probabilistic model of manufacturing tolerances are as much important as the number $N$ of blades grows.

\section{NUMERICAL EXAMPLE}

The bladed disk considered is constituted of a disk and 24 blades which are modeled by using the linear thin plane theory in bending mode. The membrane and the bending motions are fully decoupled. We are only interested in the outplane displacements. The bladed disk is located in the plane $(O x, O y)$ of a cartesian coordinate system. It should be noted that in the case of such a rotating structure, the outplane vibration response would not be affected by gyroscopic terms. The disk is made of a homogeneous and isotropic material with constant thickness $5 \times 10^{-3} \mathrm{~m}$, inner radius $3.5 \times 10^{-2} \mathrm{~m}$, outer radius $0.1 \mathrm{~m}$, mass density $7860 \mathrm{~kg} / \mathrm{m}^{3}$, Poisson ratio 0.25 and Young modulus $1.89 \times 10^{11} \mathrm{~N} / \mathrm{m}^{2}$. The inner radius of the disk is assumed to be fixed. Each blade is made of a homogeneous and isotropic material with length $7 \times 10^{-2} \mathrm{~m}$, width $8.5 \times 10^{-3} \mathrm{~m}$, linear decreasing thickness from $5 \times 10^{-3} \mathrm{~m}$ to $1 \times 10^{-3} \mathrm{~m}$, mass density $7860 \mathrm{~kg} / \mathrm{m}^{3}$, Poisson ratio 0.25 and Young modulus $2 \times 10^{11} \mathrm{~N} / \mathrm{m}^{2}$. A damping model is added for the bladed disk, corresponding to a hysteretic model with a mean loss factor $\eta=0.003$.

The mean finite element model shown in Fig. 2 is constituted of 312 (with 4 nodes) bending plate elements and has 1296 DOFs such that the DOF number of one blade is $n_{a}=36$, the DOF number of the disk is $n_{d}=576$ and the DOF number of a blade-disk coupling interface is $n_{\Sigma}=6$. Figure 3 displays the eigenfrequencies of the generalized eigenproblem associated with the tuned mechanical system in function of the circumferential wave num- 


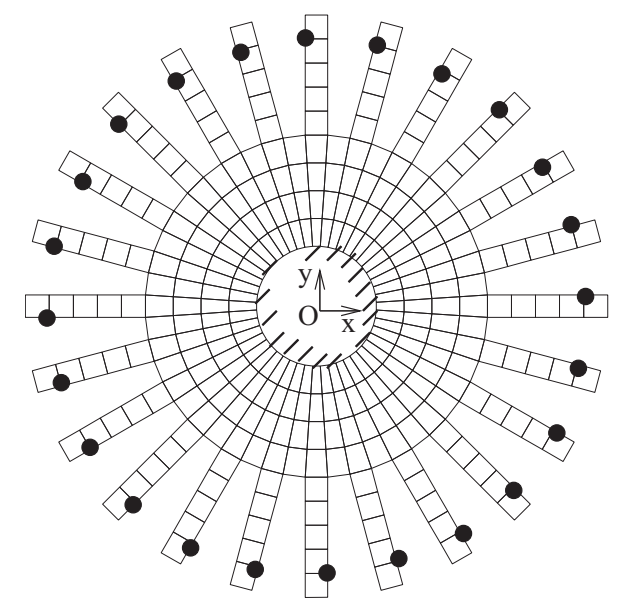

Figure 2. Finite element mesh of the bladed disk. Input force localization (symbol • )

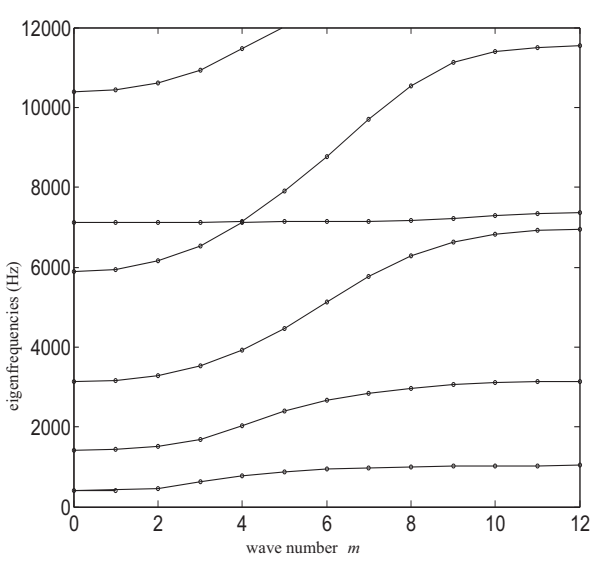

Figure 3. Graph of the eigenfrequencies values (in $\mathrm{Hz}$ ) of the tuned bladed disk versus the circumferential wave number $m$

ber $m$. A frequency "veering" [26] can clearly be seen and leads us to choose the frequency band of analysis $\mathbb{B}=[7080,7160] \mathrm{Hz}$ corresponding to a circumferential wave number $m=4$. A 4 engine order excitation is then applied to excite the modes of the veering zone. Each blade $j$ is submitted to a unit transverse load vector $\underline{\mathbf{f}}^{j}(\omega)$ such that $\underline{\mathbf{f}}^{j}(\omega)_{k}=\mathbb{1}_{\mathbb{B}}(\omega) \delta_{k,(2 d+1)} \exp (2 i \pi m j / N)$ in which $m=4$ and where $d$ is the node number of the applied force.

\section{Direct problem}

The mistuning is introduced by fixing the values $\delta_{K}^{j}=\delta_{K}$, $\delta_{M}^{j}=\delta_{M}=0, \delta_{D}^{j}=\delta_{D}=0.05$. In order to perform the mistuning analysis, we are interested in density probability function $p_{B_{\infty}}\left(b_{\infty}\right)$ and in probability $\mathcal{P}\left(B_{\infty}>b_{\infty}\right)$. The convergence analysis is then limited to the second-order convergence of the random variable $B_{\infty}$ for $N_{g}=8, \tilde{n}=120$. The norm $\left\|\left|B_{\infty} \|\right| \mid\right.$ such that ||$\left|B_{\infty}\right|||=\sqrt{\mathcal{E}\left\{B_{\infty}^{2}\right\}}$ is then estimated by

$$
||\left|B_{\infty}\right| \|^{2} \simeq \frac{1}{n_{s}} \sum_{i=1}^{n_{s}} B_{\infty}\left(\theta_{i}\right)^{2}
$$

in which $\theta_{i}$ is the realization number $i$. It can be shown that a good convergence is obtained for $n_{s}=600$. In the following, the sensitivity of the presence of uncertainties at coupling interface blade-disk are investigated. Two cases are also considered. The random reduced matrix of dynamic stiffness derived from Eq.(10) is either written as

$$
\begin{aligned}
& {\left[\mathbf{A}_{\mathrm{red}}^{j}(\omega)\right]=\left[\begin{array}{l}
{\left[\mathcal{A}^{j}(\omega)\right]\left[\mathbf{A}_{c}^{j}(\omega)\right]} \\
{\left[\mathbf{A}_{c}^{j}(\omega)\right]^{T}\left[\mathbf{A}_{\Sigma}^{j}(\omega)\right]}
\end{array}\right]} \\
& {\left[\mathbf{A}_{\mathrm{red}}^{j}(\omega)\right]=\left[\begin{array}{l}
{\left[\mathcal{A}^{j}(\omega)\right]\left[\underline{A}_{c}^{j}(\omega)\right]} \\
{\left[\underline{A}_{c}^{j}(\omega)\right]^{T}\left[\underline{A}_{\Sigma}^{j}(\omega)\right]}
\end{array}\right]}
\end{aligned}
$$

Figure 4 to 5 show the corresponding mappings $b_{\infty} \mapsto \mathcal{P}\left(B_{\infty}>\right.$ $\left.b_{\infty}\right)$ for several values of $\delta_{K}=0.01, \delta_{K}=0.08$. The thick lines are related to the nonparametric approach used with both random generalized coordinates terms and random interface whereas the thick dashed lines do only take into account the uncertainties related to random generalized coordinates. The error generated by the approximation of neglecting random uncertainties on the blade coupling interface is in general acceptable. It is also concluded that the main contribution of the response amplification comes from the generalized coordinates.

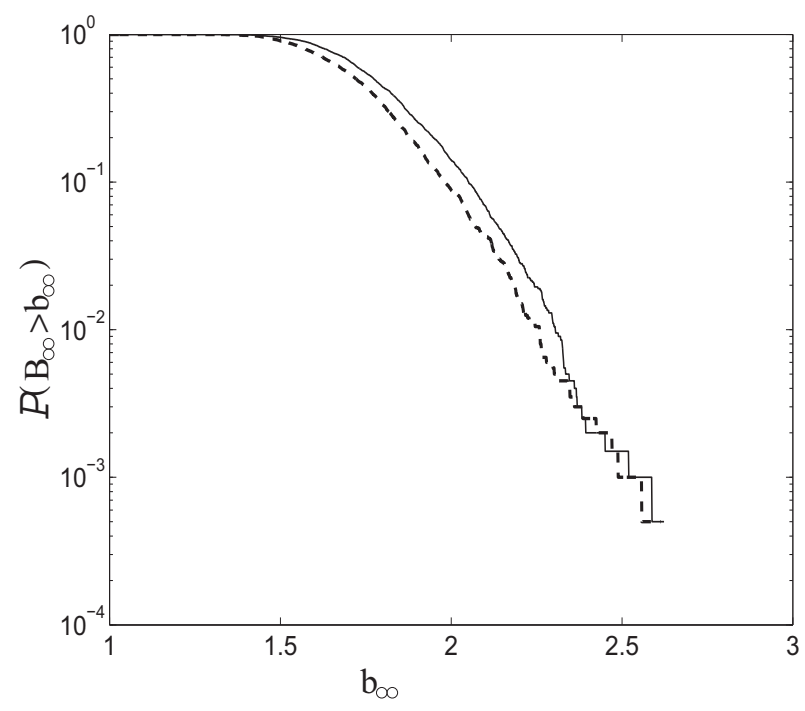

Figure 4. Influence on the blade-interface coupling for a mistuning rate $\delta_{K}=0.01$ : graph of $b_{\infty} \mapsto \mathcal{P}\left(B_{\infty}>b_{\infty}\right)$ in the case of random uncertainties on the whole reduced blade (thick line) and in the case of uncertainties on the generalized coordinates (thick dashed line) 


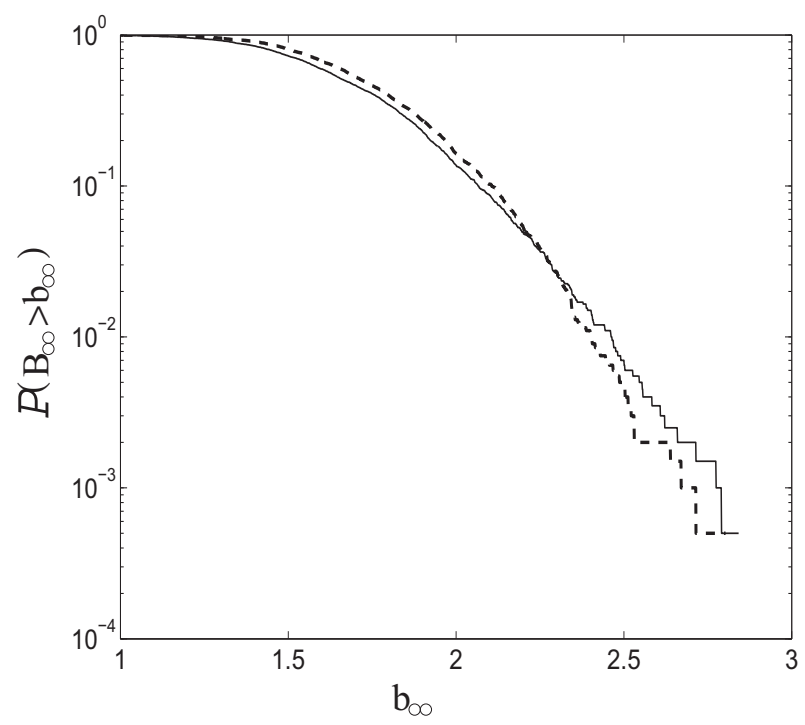

Figure 5. Influence on the blade-interface coupling for a mistuning rate $\delta_{K}=0.08$ : graph of $b_{\infty} \mapsto \mathcal{P}\left(B_{\infty}>b_{\infty}\right)$ in the case of random uncertainties on the whole reduced blade (thick line) and in the case of uncertainties on the generalized coordinates (thick dashed line)

\section{Inverse problem}

The nominal blade is modeled by a plane rectangular plate. Let $(A, x, y)$ be a cartesian system related to the blade. In this example, only manufacturing tolerances of first order are taken into account. It is supposed that the base of the blade is not affected by manufacturing tolerances. Let $\mathbf{T}=\left(\Delta \alpha_{1}, \Delta \alpha_{2}, \Delta L_{1}, \Delta L_{2}, \Delta e\right)$ be the centered random variable, where each component is independent from another and is a real centered uniform random variable. Random variable $\Delta \alpha_{1}$ ( or $\left.\Delta \alpha_{2}\right)$ characterizes the geometrical tolerances controlling the deviation angle between the upper (or lower) side of the manufactured blade and the corresponding side of the nominal blade. Random variable $\Delta L_{1}$ (or $\Delta L_{2}$ ) characterizes the dimensional tolerances allowing the length of the upper (or lower) side of the blade to fluctuate. $\Delta e$ corresponds to the thickness authorized fluctuation at the tip of the blade. It is also supposed that the thickness fluctuation over the blade length is considered linear between the base and the tip of the blade and is constant over its width. The finite element method yields 12 nodes which are all on the boundary of the blade (see Fig. 6) A probabilistic model derived from the tolerances parameters is then constructed for the nodes of the mesh and is written at first order in term of coordinates in $(A, x, y)$ :

$A M_{j}=A m_{j}+\frac{j-1}{5}\left(\Delta x_{1}, \Delta y_{1}\right), \Delta z_{j}=\frac{j-1}{5} \Delta e, j \in\{1, \ldots, 6\}$

$A M_{j}=A m_{j}+\frac{j-7}{5}\left(\Delta x_{2}, \Delta y_{2}\right), \Delta z_{j}=\frac{j-7}{5} \Delta e, j \in\{7, \ldots, 12\}$

where $M_{j}$ and $m_{j}$ denote the nodes of the mesh related to the random blade and the nominal blade respectively and where
$\Delta x_{1}=\Delta L_{1}, \Delta x_{2}=\Delta L_{2}, \Delta y_{1}=L \Delta \alpha_{1}, \Delta y_{2}=L \Delta \alpha_{2}$ and $\Delta e$ are the centered uniform random variables such that the corresponding standard deviations verify

$$
\sigma_{\Delta x_{1}}=\sigma_{\Delta x_{2}}=\sigma_{\Delta y_{1}}=\sigma_{\Delta y_{2}}=\sigma_{\Delta e}=\frac{\tau}{\sqrt{3}}
$$
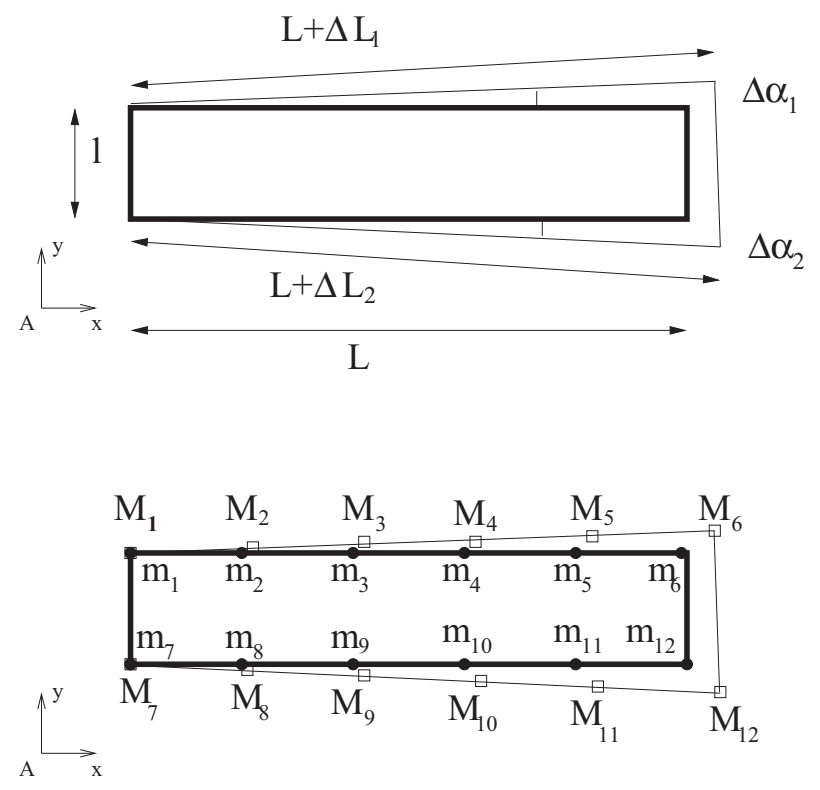

Figure 6. Nominal blade (thick line) and manufactured blade (thin line)

The parameter $\tau$ characterizes the upper bound of the fluctuations. A parametric study is then carried out with $\tau \in \mathbb{T}=$ $[0.1,100] \times 10^{-6} \mathrm{~m}$. Concerning the first Monte Carlo numerical simulation allowing the dispersion levels $\delta_{M}$ and $\delta_{K}$ to be known, a convergence analysis is performed with respect to $n_{t}$ realizations denoted by $\theta_{1}, \ldots, \theta_{n_{t}}$. For a fixed $\tau \in \mathbb{T}$ and for each $\theta_{j}$, the quantity $\left\|\left[B_{\text {red }}\left(T\left(\theta_{j}\right)\right)\right]-\left[\underline{B}_{\text {red }}\right]\right\|_{F}^{2}, B \in\{M, K\}$ is calculated. Parameter $\delta_{B}$ is then estimated by $\hat{\delta}_{B}\left(n_{t}\right)$ such that

$$
\hat{\delta}_{B}\left(n_{t}\right) \simeq \sqrt{\frac{\left(N_{a}^{+}+1\right) \frac{1}{n_{t}} \sum_{j=1}^{n_{t}}\left\|\left[B_{\mathrm{red}}\left(T\left(\theta_{j}\right)\right)\right]-\left[\underline{B}_{\mathrm{red}}\right]\right\|_{F}^{2}}{\operatorname{tr}\left(\left[\underline{B}_{\mathrm{red}}\right]\right)^{2}+\operatorname{tr}\left(\left[\underline{B}_{\mathrm{red}}\right]^{2}\right)}} .
$$

Figure 7 shows the graph $n_{t} \mapsto \hat{\delta}_{M}\left(n_{t}\right)$ (thick line) and $n_{t} \mapsto \hat{\delta}_{K}\left(n_{t}\right)$ (thick dashed line) for $\tau=100 \times 10^{-6} \mathrm{~m}$. A good convergence is then obtained for $n_{t}=600$. In the following, parameters $\delta_{M}$ and $\delta_{K}$ denote $\hat{\delta}_{M}\left(n_{t}=600\right)$ and $\hat{\delta}_{K}\left(n_{t}=600\right)$. Figure 8 shows the graphs of $\tau \mapsto \delta_{K}$ and $\tau \mapsto \delta_{M}$ which allow the dispersion of the random reduced matrices issued for the a priori probabilistic model to be estimated with respect to parameter $\tau$ characterizing the tolerance rate. It is clearly seen that $\delta_{K}$ and 


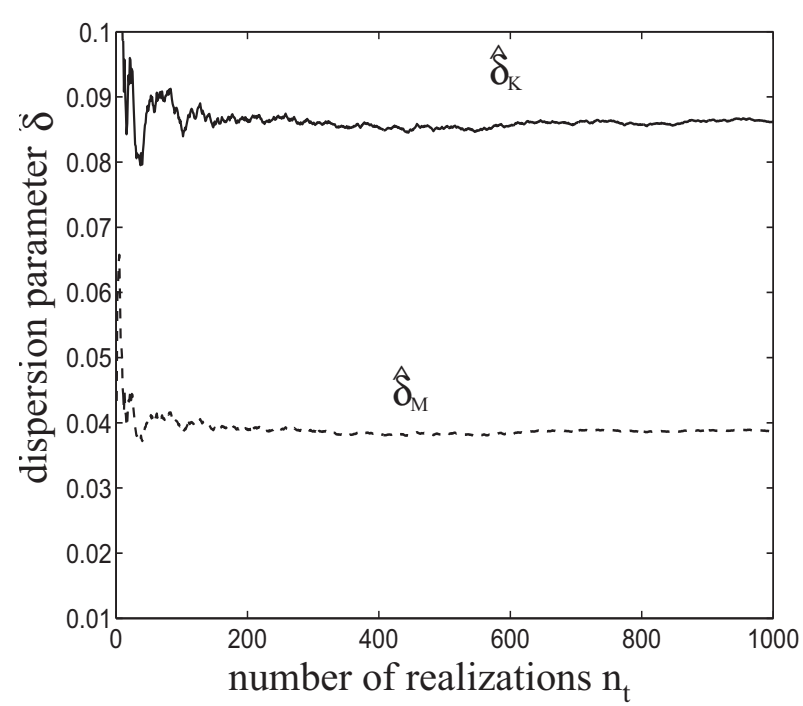

Figure 7. Convergence analysis : graph of $n_{t} \mapsto \hat{\delta}_{M}\left(n_{t}\right)$ (thick line) and $n_{t} \mapsto \hat{\delta}_{K}\left(n_{t}\right)$ (thick dashed line) for the tolerancing rate $\tau=100 \times$ $10^{-6} \mathrm{~m}$

$\delta_{M}$ are not independent and that there are proportional to parameter $\tau$. The dispersion parameter related to the mass is lower than the dispersion parameter related to the stiffness. In the present context, when considering the mistuning problem of the whole bladed-disk, $\delta_{M}$ and $\delta_{K}$ constitute the data of the nonparametric probabilistic model. It is assumed that the uncertainties related to each blade are homogeneous from one blade to another one such that $\delta_{M}^{j}=\delta_{M}$ and $\delta_{K}^{j}=\delta_{K}$ for $j \in\{0, \ldots, N-1\}$. Although the geometrical uncertainties of a blade create many uncertainty on the blade mass matrix, we are interested by studying the mistuning effects of the assembled bladed-disk. From a technological point of view the bladed-disk is dynamically balanced by adding or removing material on the disk during the process of assemblage. Consequently, we assume that the uncertainty on the mass has been sufficiently reduced by the balancing process in order to neglect it such that $\delta_{M}=0$. Furthermore, we assume that the modifications of the disk have a small impact on the random dynamical magnification factor and are neglected.

A mistuning analysis is then performed for the complete structure using the direct problem described above. An uncertainty on the damping is fixed by $\delta_{D}=\delta_{D}^{j}=0.05$. We are interested in studying $\mathcal{P}\left(B_{\infty}>b_{\infty}\right) \leq p$ where $b_{\infty}$ denotes the magnification level over the frequency band of analysis $\mathbb{B}$ and where $p$ denotes a probability level. The criterion which assesses the correct work of the bladed-disk is defined by parameters $\left(b_{\infty}, p\right)$. Figure 9 shows the graph $\delta_{K} \mapsto \mathcal{P}\left(B_{\infty}>b_{\infty}\right)$ for several values $b_{\infty}=1.2$ (thin dashed-dotted line), $b_{\infty}=1.3$, (thin dashed line) $b_{\infty}=1.4$ (thin line), $b_{\infty}=1.5$ (thick dashed-dotted line), $b_{\infty}=1.6$ (thick dashed line) and $b_{\infty}=1.7$ (thick line). For a given $p$, the domain of values of the appropriate dispersion parameter $\delta_{K}$ characterizing the amount of mistuning can be deduced from

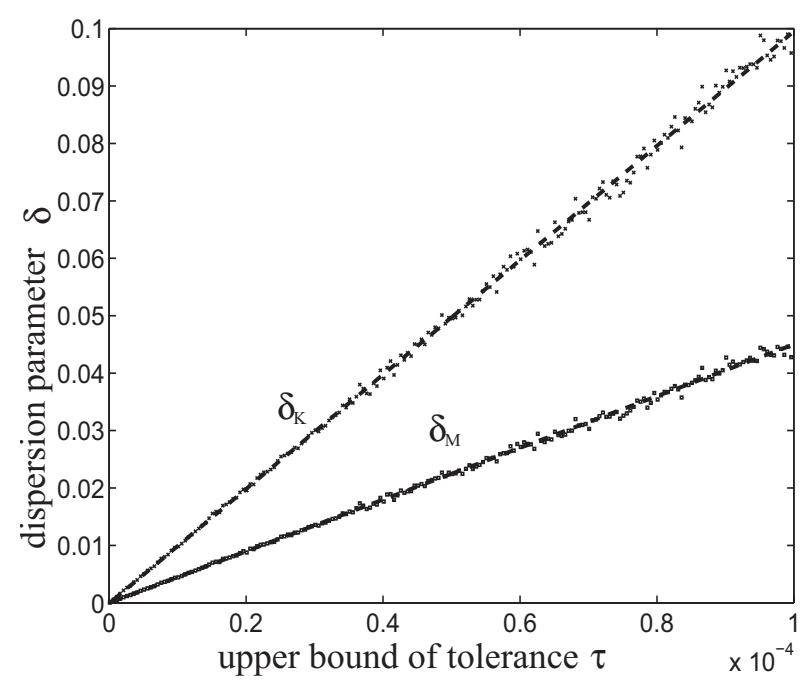

Figure 8. Tolerancing analysis: Graph of $\tau \mapsto \delta_{M}$ and $\tau \mapsto \delta_{K}$ allowing the dispersion of random matrices issued from the a priori probabilistic model to be characterized.

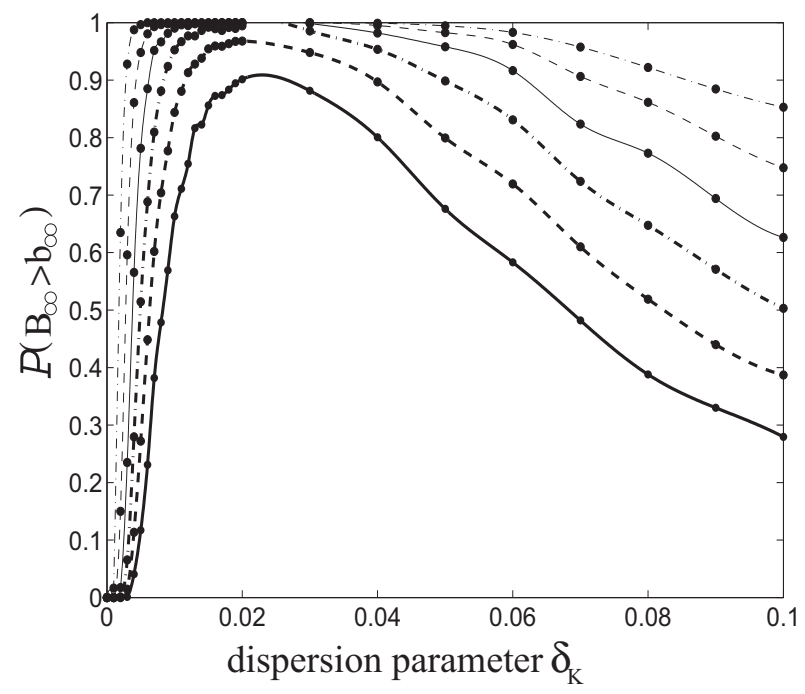

Figure 9. Mistuning analysis: Graph of $\delta_{K} \mapsto \mathcal{P}\left(B_{\infty}>b_{\infty}\right)$ for dispersion parameters $\delta_{M}=0, \delta_{D}=0.05$ and for several values of $b_{\infty}$ : $b_{\infty}=1.2$ (thin dash-dotted line), $b_{\infty}=1.3$ (thin dashed line), $b_{\infty}=1.4$ (thin line), $b_{\infty}=1.5$ (thick dash-dotted line), $b_{\infty}=1.6$ (thick dashed line), $b_{\infty}=1.7$ (thick line).

Fig. 9. The corresponding upper bound of tolerances are directly read from Fig 8. For example, if the quality of a bladed disk is defined by $\left(b_{\infty}=1.5, p=0.6\right)$, it can be seen on Fig.9 that the confidence region defined by $\mathcal{P}\left(B_{\infty}>1.5\right) \leq 0.6$ corresponds to the following value of dispersion parameters $\delta_{K} \leq 0.0041$ or $\delta_{K} \geq 0.0862$. From Fig. 8 , it can then be deduced that the tolerance should be such that $\tau \leq 4.1 \mu \mathrm{m}$ or $\tau \geq 86 \mu \mathrm{m}$. The global maximum of function $\delta_{K} \mapsto \mathcal{P}\left(B_{\infty}>b_{\infty}\right)$ is of importance 
because it shows that there exists a forbidden band of values $\left[\tau_{\min }, \tau_{\max }\right]$ for the upper bound of tolerances $\tau$. From $\tau<\tau_{\min }$, it is deduced that the blade has to be manufactured with a high precision to assess the requirement. If $\tau>\tau_{\max }$, the requirement is verified too. There are also two ways for specifying tolerances, one which requires a higher precision during the manufacturing process, another one which is related to intentional mistuning. It is also shown that a manufactured blade, which is intentionally mistuned by specifying an upper bound such that $\tau>\tau_{\max }$ or which is manufactured with accurate specifications of tolerances such that $\tau<\tau_{\min }$, can present the same characteristics concerning the random dynamical magnification factor related to the forced response of the bladed disk.

\section{CONCLUSION}

In this paper, a nonparametric model of random uncertainties has been proposed to analyze the mistuned bladed-disks. This new approach is based on the use of a probability model which has the largest entropy among all probability models satisfying the constraints due to the available information. In particular, contrary to the gaussian models used in the literature, the proposed probabilistic model guarantees the random matrices to be positive-definite, which is absolutely necessary for such a dynamical problem. Furthermore, this new approach differs from the usual parametric model in the sense that it is able to represent not only the blade eigenfrequencies uncertainties but also the eigenmodes uncertainties. It should be noted that any probability model of random uncertainties has to be consistent with respect to the perturbation of the eigenvalues and the eigenvectors. Consequently, there is no meaning to treat separately the perturbations of the eigenfrequencies and the perturbation of the eigenmodes. The nonparametric approach is a global approach and both eigenmodes and eigenfrequencies uncertainties are quantified by only three scalar parameters related to mass, damping and stiffness matrices. Moreover, an inverse methodology using this nonparametric approach has been proposed in order to specify the manufacturing tolerances with respect to the confidence region of the random dynamical magnification factor over a narrow frequency band of analysis. The efficiency of the method has been shown through a numerical example. It has been outlined that the presence of random uncertainties on the coupling interface do not play a significant role with respect to the mistuning analysis. Thanks to a parametric analysis with respect to the dispersion parameter, the characteristics of tolerancing are easily specified from a criterion concerning the dynamical magnification factor.

\section{BIBLIOGRAPHY}

[1] Thomas, D.L., 1979, "Dynamics of Rotationally Periodic Structures", International Journal for Numerical Methods in Engineering, 14, pp.81-102.

[2] Bossavit, A., 1985, "Boundary Value Problems with Symmetry and their Approximation by Finite Elements", SIAM
Journal of Applied Mathematics, 53(5), pp.1352-1380.

[3] Ohayon, R. and Soize, C., 1998, Structural Dynamics and Vibration, London, Academic Press.

[4] Irwanto, B., Hardtke, H.J., Pawandenat, D. and Schmidt, R., 2001, "On Substructuring Technique: Application of Lagrange Multiplier Method on Cyclic Structures", Journal of Sound and Vibration, 247(5), pp.939-945.

[5] Whitehead, D.S., 1966, "Effects of Mistuning on the Vibration of Turbomachine Blades induced by Wakes", Journal of Mechanical Engineering Science, 8(1), pp.15-21.

[6] Dye, R.C.F. and Henry, T.A., 1969, "Vibration Amplitudes of Compressor Blades Resulting from Scatter in Blade Natural Frequencies", ASME Journal of Engineering for Power, 91(3), pp.182-187.

[7] Ewins, D.J., 1969, "The Effects of Detuning upon the Forced Vibrations of Bladed Disks", Journal of Sound and Vibration, 9(1), pp.65-69.

[8] Wei, S.T. and Pierre, C., 1988, "Localization Phenomena in Mistuned Assemblies for Cyclic Symmetry - Part II: Forced Vibrations", ASME Journal of Vibration, Acoustics, Stress, and Reliability in Design, 110(4), pp.439-449.

[9] Lin, C.C. and Mignolet, M.P. , 1997, "An Adaptative Perturbation Scheme for the Analysis of Mistuned Bladed Disks", ASME Journal of Engineering for Gas Turbines and Power, 119(1), pp.153-160.

[10] Mignolet, M.P. and Lin, C.C., 2001, "A Novel Limit Distribution for the Analysis of Randomly Mistuned Bladed Disks", ASME Journal of Engineering for Gas Turbines and Power, 123(2), pp.388-394.

[11] Griffin, J.H. and Hoosac, T.M., 1984, "Model Development and Statistical Investigation of Turbine Blade Mistuning", ASME Journal of Vibration, Acoustics, Stress, and Reliability in Design, 106(2), pp.204-210.

[12] Sinha, A. and Chen, S., 1989, "A Higher Order Technique to Compute the Statistics of Forced Response of a Mistuned Bladed Disk Assembly", Journal of Sound and Vibration, 130(2), pp.207-221.

[13] Yang, M-T. and Griffin, J.H., 2001, ”A Reduced-Order Model of Mistuning Using a Subset of Nominal Modes", ASME Journal of Engineering for Gas Turbines and Power, 123(3), pp.893-900.

[14] Bladh, R., Castanier, M.P. and Pierre, C., 2001, "Component-Mode-Based Reduced order Modeling Techniques for Mistuned Bladed Disks-Part 1: Theoretical Models", ASME Journal of Engineering for Gas Turbines and Power, 123(1), pp.89-99.

[15] Seinturier, E., Dupont, C., Berthillier, M. and Dumas, M., 2002, "A New Aeroelastic Model for Mistuned Bladed Disks", AIAA Journal, AIAA-2002 - 1533 (accepted for publication)

[16] Mignolet, M.P., Rivas-Guerra, A.J. and Delor, J.P., 2001, "Identification of Mistuning Characteristics of Bladed Disks From Free Response Data (Part 1)", ASME Journal of Engineering for Gas Turbines and Power, 123(2), pp.395-403. 
[17] Rivas-Guerra, A.J., Mignolet, M.P., and Delor, J.P., 2001, "Identification of Mistuning Characteristics of Bladed Disks From Free Response Data (Part 2)", ASME Journal of Engineering for Gas Turbines and Power, 123(2), pp.404-411.

[18] Capiez-Lernout, E., Soize, C. "Une approche probabiliste non paramétrique du phénomène de désaccordage des structures à géométrie cyclique. Séminaire Descartes du Polytechnicum de Marne-La-Vallée, 13 juin 2002, Actes des journées jeunes chercheurs, pp. 65-74.

[19] Rivas-Guerra, A.J., Mignolet, M.P., ’Maximum Amplification of Blade Response due to Mistuning: Localization and Mode Shapes Aspect of the Worst Disks", Proceedings of the ASME Turbo Expo 2002, June 3 - 6 2002, Amsterdam.

[20] Soize, C., 2000, "A Nonparametric Model of Random Uncertainties for Reduced Matrix Models in Structural Dynamics”, Probabilistic Engineering Mechanics, 15(3), pp.277-294.

[21] Soize, C., 2001, "Maximum Entropy Approach for Modeling Random Uncertainties in Transient Elastodynamics", Journal of the Acoustical Society of America, 109(5), pp.19791996.

[22] Sanliturk, K.Y., Imregun,M. 1994, "Vibration analysis of Mistuned Bladed-Disk Assemblies - Inverse Approach", AIAA Journal, 32(4), pp.857-865.

[23] Craig, R.R.Jr. and Bampton, M.C.C., 1968, "Coupling of Substructures for Dynamic Analyses", AIAA Journal, 6(7), pp.1313-1319.

[24] Soize,C. , 2001, "Nonlinear Dynamical Systems with Nonparametric Model of Random Uncertainties", Uncertainties in Engineering Mechanics, 1(1), pp.1-38, E-journal from Resonance Publication (http://www.resonancepub.com)

[25] Soize, C. and Chebli, H., 2002, "Random Uncertainties Model in Dynamic Substructuring Using a Non-Parametric Probabilistic Model", ASCE Journal of Engineering Mechanics, (accepted for publication)

[26] Pierre, C.,1988, "Mode Localization and Eigenvalue Loci Veering Phenomena in Disordered Structures", Journal of Sound and Vibration, 126(3), pp.485-502. 